\title{
INFLUENCIA DE HERBICIDAS NA NODULAÇÃO DA SOJA (Glycine max L. Merr.)
}

A.R. Giardini (1); E.S. Lopes ( $\left.{ }^{1}\right)$ \& R. Deuber (2)

(') Seção de Microbiologia do Solo

$\left(^{2}\right)$ Seção de Fisiologia

Pesquisadores Científicos, Instituto Agronômi co, Caixa Postal 28, 13.100 Campinas,Est. S. Paulo. Bolsistas do CNPq.

Trabalho apresentado no «Simpósiou Internacional sobre limitações e potenciais da fixação simbiótica de $\mathrm{N}$ nos trópicos", em Brasilia, julho de 1977.

Recebido para publicação em 10.3.1979

\section{RESUMO}

Um experimento foi conduzido em Campinas, em latossolo vermelho escuro, barrento, por dois anos consecutivos, para testar o efeito dos herbicidas trifluralin, vernolate e alachlor e duas doses de nitrogênio, na nodulação e produção de soja, CV santa rosa, inoculada. 
No primeiro ano, os herbicidas não influenciaram a nodulação aos 35, 58 e 98 dias após o plantio. A aplicação de nitrogênio diminuiu o peso dos nódulos secos observados aos 58 dias, e seu número e peso, aos 98 dias. A produção de grãos foi semelhante para todos os tratamentos.

No segundo ano, a nodulação das plantas inoculadas não foi influenciada pela aplicação de nitrogênio, porém nos tratamentos co m nodulação espontânea (não inoculados), o peso dos nódulos secos diminuiu significativamente, nas três épocas, devido à aplicação daquele nutriente. Os tratamentos inoculados produziram maiores quantidades de sementes em comparação com os tratamentos não inoculados. Houve interação significativa de inoculação $\mathrm{x}$ adubação nitrogenada, sendo que o aumento devido à adubação só foi verificado no tratamento sem inoculação. Nos canteiros em que se fez a aplicação de herbicidas, a produção de grãos foi maior; não foi, entretanto, possivel atribuir esse efeito à aplicação dos herbicidas.

UNITERMOS: herbicidas, nodulação, soja.

\section{SUMMARY}

INFLUENCE OF HERBICIDES ON NODULATION OF SOYBEANS (Glycine max L. Merril)

A field trial was performed on a loamy soil, for two consecutive years, to study the effect of trifluralin, vernolate and alachlor and two rates of nitrogen, on nodulation and yield of inoculated soybeans.

The herbicides did not affect the nodulation or yield during the crop cycle, in any of the experiments. The $\mathrm{N}$ application depressed the weight of dry nodules at 58 days and its number and weight at 98 days in the first year. The yield of the different treatmens showed no difference.

In the second year the nitrogen application did not affect the nodulation of inoculated plants, but the weight of dry nodules of the treatments with spontaneous nodulation was significantly reduced in the three periods of sampling. This year the inoculated treatments showed higher yields when compared with those not inoculated, and the herbicide treated plots yielded more than the checks without herbicide. KEYWORDS: Herbicides, nodulation, soybeans .

\section{INT RODUÇÃO}

Para a recomendação racional do controle químico do mato, é necessário conhecer-se, além das informações sobre a fitotoxicidade do produto à cultura, eficiência no controle do mato e as- pectos econômicos da sua aplicação, os possiveis efeitos colaterais benéficos, ou deletérios, dos herbicidas na atividade microbiana do solo, ou, pelo menos, na atividade de determinados microrganismos que têm implicacões diretas no processo de produção. Efeitos benéficos na fixação de nitrogênio por Spirillum lipoferum foram constatados em meio de cultivo, por adição de alachlor

e atrazine ao meio; raízes de milho cultivado em solo onde se fez a aplicação dos mesmos herbicidas apresentaram maior atividade da nitrogenase (9).

A soja vem sendo extensivamente cultivada no Estado de São Paulo, e o uso de herbicidas para o controle do mato, nessa cultura, aumenta a cada ano.

A maior parte das sementes de soja plantada no Estado de São Paulo é .inoculada, não se recomendando a adubação nitrogenada da cultura. É, portanto, de grande interesse conhecer-se a influên cia dos herbicidas na nodulação

e fixação de nitrogênio nesta leguminosa.

Poucos trabalhos foram feitos em nosso meio para estudar a influência de herbicidas na nodulação de leguminosas. Lopes et al. (5) observaram que trifluralin e EPTC não prejudicaram a nodulação do feijoeiro inoculado, no campo. Tampouco o EPTC causou qualquer alteração na nodulação natural de feijão (3).

Lorenzi e Araujo (8), estudando o efeito de vários herbicidas na nodulação da soja, cultivar Davis, observaram que trifluralin e alachlor não influen ciaram a nodulação causada pela estirpe inoculada à semente, porém o trifluralin afetou a nodulação causada por bactérias presentes no solo.

Em experimentos conduzidos em vasos, Kust e Struckmeyer (4) verificaram uma diminuição no crescimento da planta e na nodulação da soja devido à aplicação de trifluralin; aparentemente, o herbicida diminuiu a translocação das reservas cotiledonares. 
O objetivo do presente trabalho foi de estudar, em condições de campo, a influência dos herbicidas trifluralin, vernolate e alachlor, na nodulação e produção de grãos de soja inoculada, em presença e ausência da adubação nitrogenada.

\section{MATERIAL E MÉTODOS}

O experimento foi instalado nos anos de 1973 e 1974, no Centro Experimental de Campinas, em latossolo vermelho escuro, série Barão (10) em área des provida de Rhizobium japonicum (7).

A análise granulométrica do solo revelou os seguintes resultados: $35 \%$ de argila, $7,5 \%$ de limo, $27,3 \%$ de areia fina e $30,2 \%$ de areia grossa; a análise química apresentou os seguintes resultados: $\mathrm{pH} 5,66 ; 3,4 \%$ de matéria orgânica; 0,08 de $\mathrm{PO} 4-3 ; 0,1 \mathrm{de} \mathrm{K}^{+} \mathrm{e}$ 2,04 de $\mathrm{Ca}+\mathrm{Mg}^{++}$e.mg/100 ml de T.F.S.A.

Um mês antes do primeiro plantio fez-se a calagem, incorporando-se o equivalente à $2.000 \mathrm{~kg} /$ ha de calcário dolomítico em toda área experimental. A adubação básica foi também feita apenas no primeiro ano, com superfosfato simples (100 kg P205/ha) e com cloreto de potássio (60 kg K20/ha), sendo que os adubos foram aplicados no sulco de plantio, ao lado e abaixo das sementes.

O plantio, nos dois anos, foi feito no dia 04 de dezembro, usando-se o cultivar santa rosa, que é o mais cultivado no Estado de São Paulo. As sementes foram desinfetadas com brometo de metila, antes do plantio. O inoculante utilizado foi preparado usando-se uma mistura das estirpes SMS-65 e SMS-313, eficientes para o cultivar santa rosa (6) e continha número satisfatório de células viáveis (acima de $7,5 \times 10^{7}$ bactérias/g) nas inoculações efetuadas em ambos os anos. A inoculação, nos tratamentos indicados, foi feita com $1 \mathrm{~g}$ do inoculaste/ $100 \mathrm{~g}$ sementes. Quinze dias após as germinações, foi feito o desbaste, deixando-se vinte plantas por metro, nos dois anos do ensaio.

O delineamento experimental foi um fatorial $4 \times 2 \times 2$, para herbicida (sem herbicida, trifluralin, vernolate e alachlor), inoculação (com e sem) e nitrogênio (com e sem), em blocos ao acaso, com cinco repetições.

Os herbicidas trifluralin e vernolate foram incorporados ao solo, a uma profundidade de 8 a $10 \mathrm{~cm}$, nas doses de 0,72 e 2,55 kg de i.a./ha, respectivamente. As aplicações desses herbicidas foram efetuadas 18 dias antes do plantio, no primeiro ano, e 10 dias antes, no segundo ano. O alachlor foi aplicado em superfície, um dia depois do plantio, na dose de $1,68 \mathrm{~kg}$ de i.a./ha nos dois anos. Utilizou-se um pulverizador de pressão constante de $2,81 \mathrm{~kg} / \mathrm{cm}^{2}$, gastando-se 400 litros de água por hectare. Nos tratamentos sem herbicida, foi feita a capina do mato, quando necessária.

O nitrogênio foi aplicado parceladamente na forma de uréia, cada 20 dias após a germinação. No primeiro ano, a adubação nitrogenada foi de 60 $\mathrm{kg}$ de N/ha em duas aplicações; no segundo ano, a dose foi aumentada para $90 \mathrm{~kg}$ de N/ha, em três aplicações.

Nos dois anos de condução dos experimentos, foram feitas três coletas de nódulos, para determinações de número e peso, arrancando-se, cuidadosamente, 10 plantas de cada parcela. Essas amostragens foram feitas aos 35, 58 e 98 dias após o plantio, no primeiro ano, e aos 46, 70 e 104, no segundo.

No primeiro ano, a colheita foi feita aos 152 dias após o plantio, e no segundo, aos 161. Foram colhidas as plantas existentes em quatro metros de cada uma das linhas centrais, contando-se as plantas colhidas.

\section{RESULTADOS E DISCUSSÃO}

A) Influência dos tratamentos na nodulação

No primeiro ano do experimento, todas as plantas dos tratamentos não 
inoculados estavam desprovidas de nódulos, conforme esperado, vis to que em experimento anterior se constatara ausência de Rhizobium japonicum em amostras de solo da área experimental (7). Para o estudo da influên cia dos her bicidas e de nitrogênio na nodulação, foram, portanto, considerados apenas os tratamentos inoculados. Nesses tratamentos houve $100 \%$ de plantas noduladas; a evolução da massa de nódulos nas três amostragens, nos tratamentos sem e com herbicida está apresentada nas figuras 1 e 2, para o primeiro e segundo ano, respectivamente. Para a amostragem de nódulos efetuada aos 58 dias, no primeiro ano, a média do número de nódulos por planta para os diferentes tratamentos com herbicidas foram as seguintes: testemunha 10,3 ; trifluralin, 9,1; vernolate 8,2 ; alachlor 8,8 . Os pesos dos nódulos secos nessa amos-

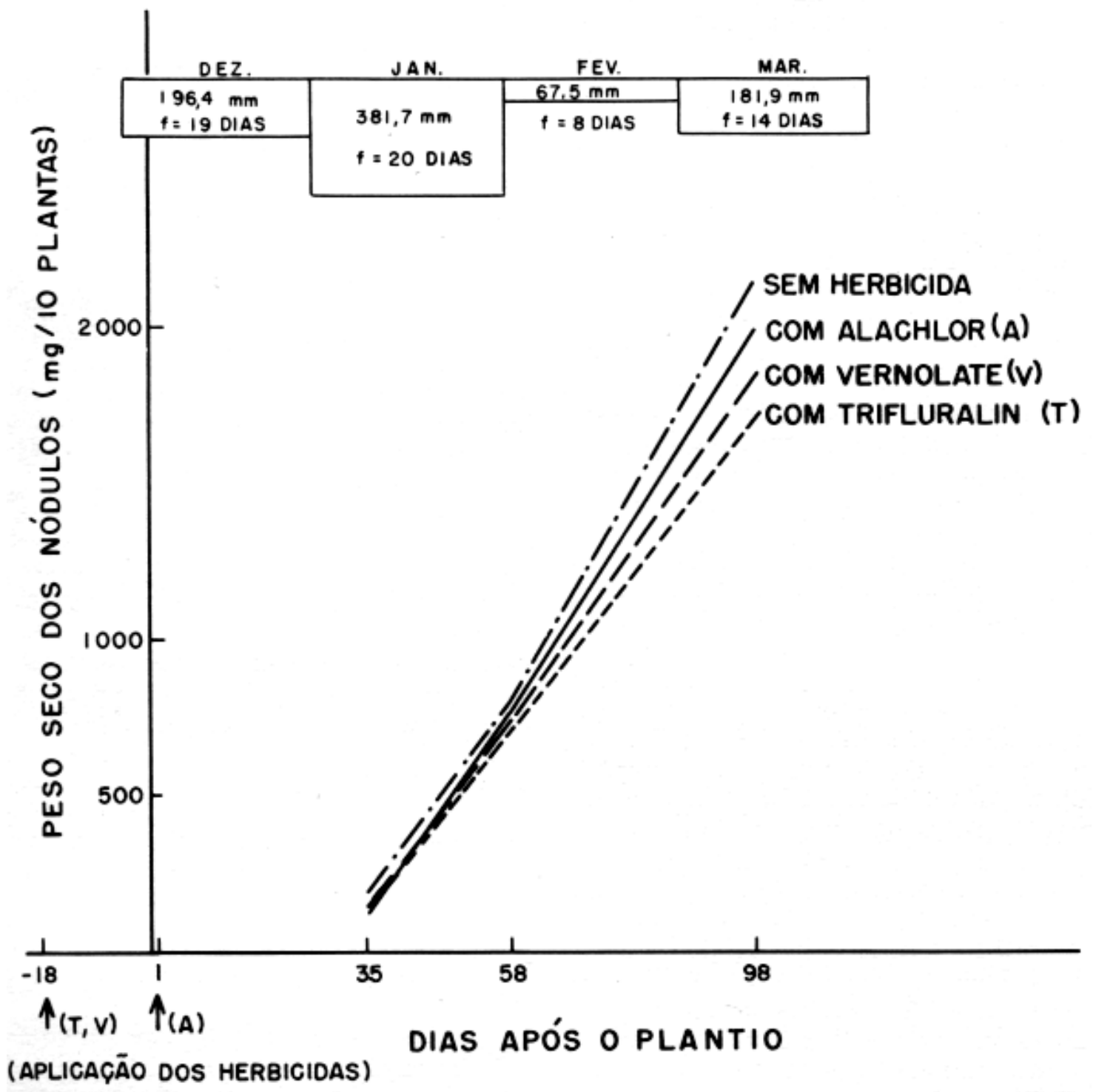

Figura 1. Influência de herbicidas na nodulação da soja inoculada. Ano agrícola - 1973/1974. 


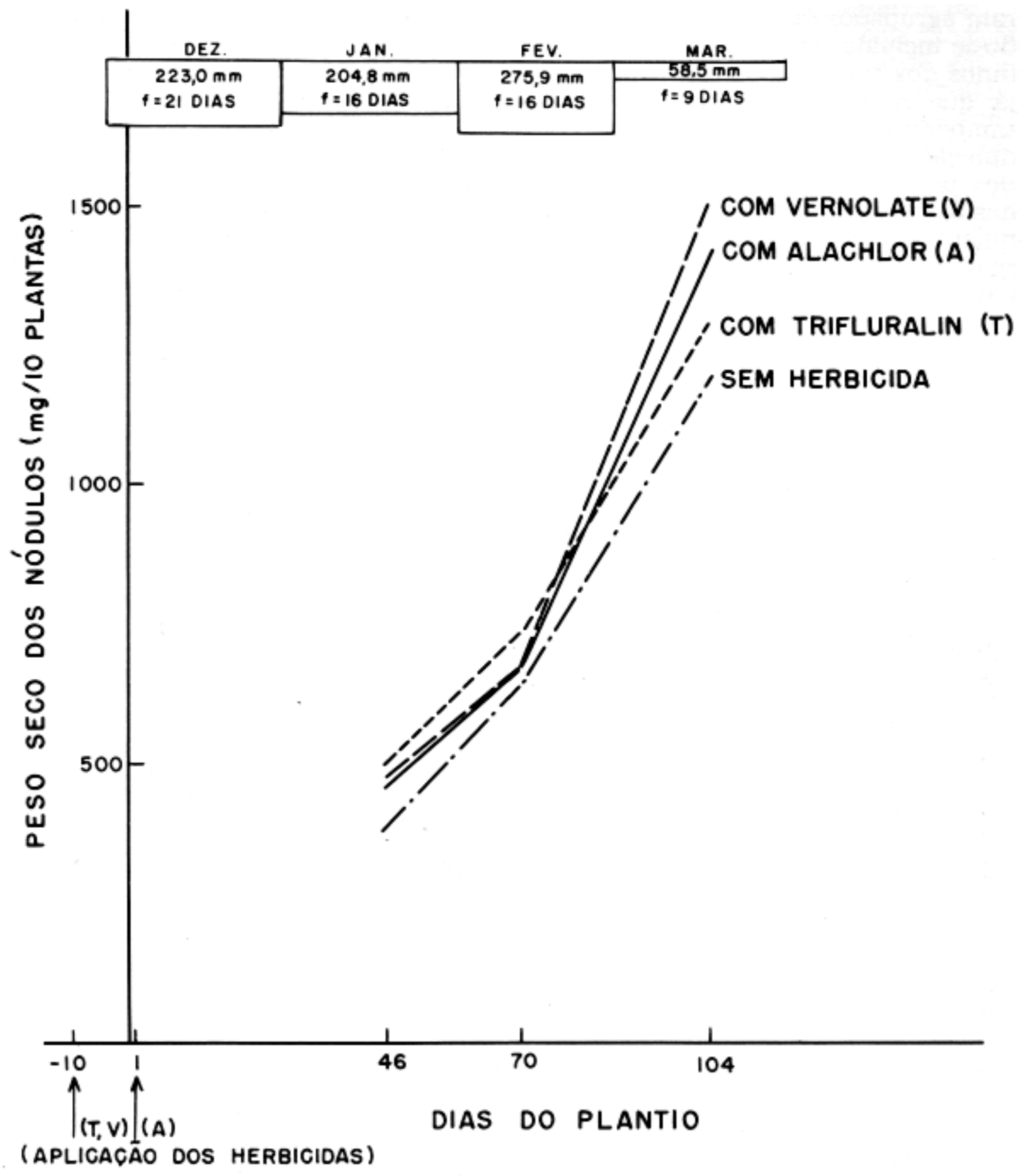

Figura 2. Influência de herbicidas na nodulaçáo da soja inoculada. Ano agrícola - 1974/1975.

tragem, na mesma ordem, foram os seguintes: 80,$9 ; 71,9 ; 74,1$ e 75,3 $\mathrm{mg} / \mathrm{planta}$. A análise estatística dos dados transformados para $\mathrm{Vx}+1$ revelou que nenhum dos herbicidas teve qualquer efei- to significativo no peso dos nódulos amostrados em três épocas, quer no primeiro, ou no segundo ano. Na figura 3 os dados de nodulação referentes aos tratamentos dos diversos herbicidas fo- 
ram agrupados numa única curva, tendo-se incluído também os dados de nódulos dos tratamentos não inoculados, já que neste ano, estes tratamentos também apresentaram nódulos. A nodulação dos tratamentos não inoculados poderia ser atribuida à dispersão das bactérias que persistiram dos tratamentos inoculados no ano anterior, porém não parece ter sido esse o caso, como se discutirá adiante.
$\mathrm{Na}$ amostragem do segundo ano, efetuada aos 70 dias após o plantio, as médias dos números de nódulos por planta foram 15,0 para testemunha, 18,4 para o trifluralin, 14,3 para vernolate e 14,7 para o alachlor. As médias de peso dos nódulos secos, na mesma ordem foram: 64,4; 73,6; 67,0 e 66,6 mg/planta.

A ausência de prejuízo do trifluralin (e também EPTC) na nodulação do feijoeiro foi anteriormente relatada por

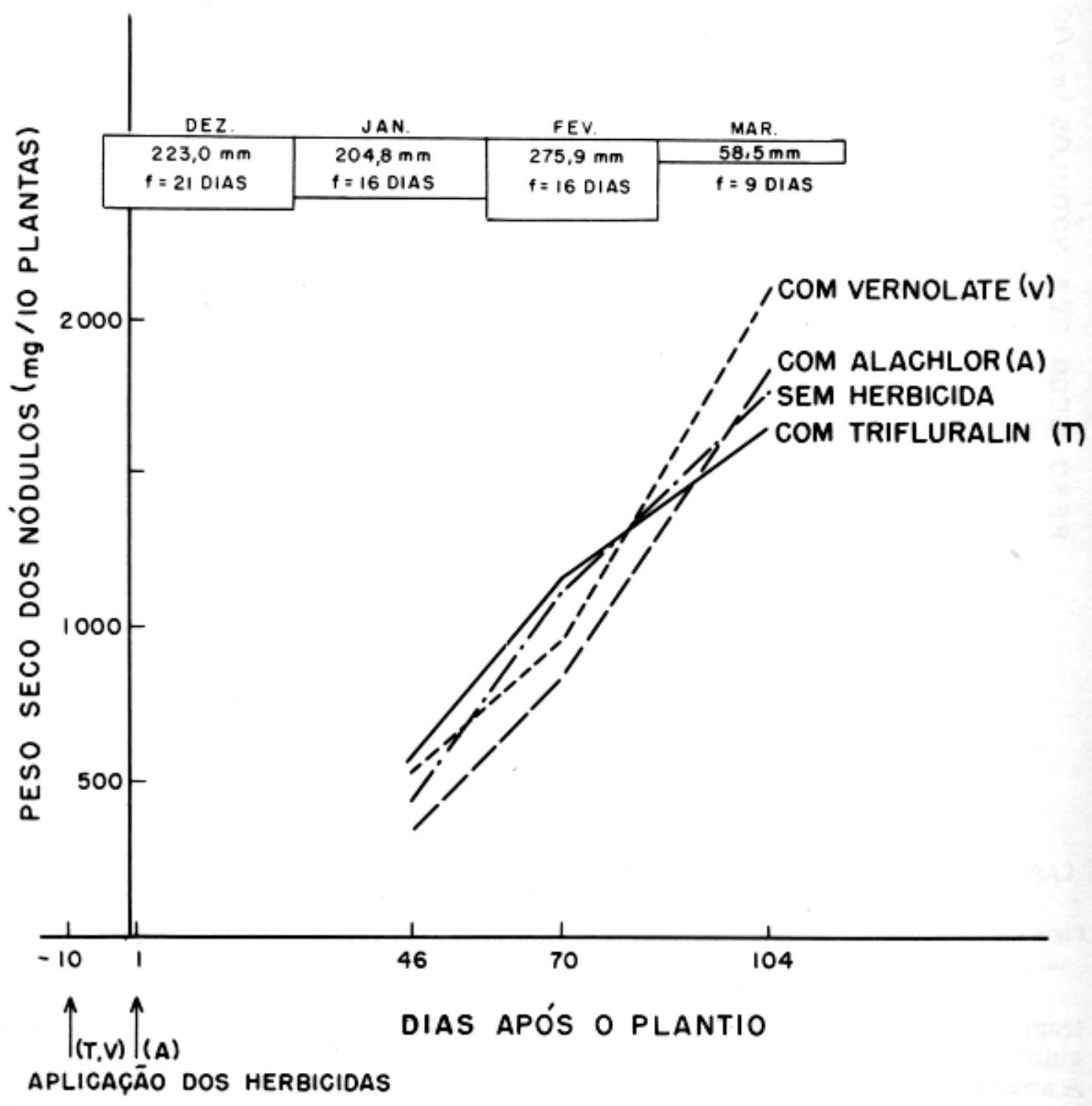

Figura 3. Influência de herbicidas na nodulação da soja não inoculada. Ano agrícola - 1974/1975. 
Lopes et al. (5). No trabalho de Lorenzi e Araujo (8) há indicações de que a influência dos herbicidas pode variar com a estirpe de Rhizobium, vis to que enquanto não houve influência nos canteiros inoculados, nos tratamentos não inoculados, que formaram nódulos devido a rizóbios nativos, houve redução na nodulação pelos herbicidas.

Parker e Dowler (11), usando trifluralin e vernolate, em dosagens com- paradas às deste ensaio, observaram diminuição da nodulação da soja causada pelo trifluralin, não observando entretanto efeito prejudicial do vernolate, que nas dosagens mais elevadas apresentou tendência para aumento da nodulação.

Existem indicações de que o trifluralin, em concentrações de 0,25 ppm em solução nutritiva agarificada retarda a nodulação do trevo subterrâneo. Caso um retardamento de nodulação

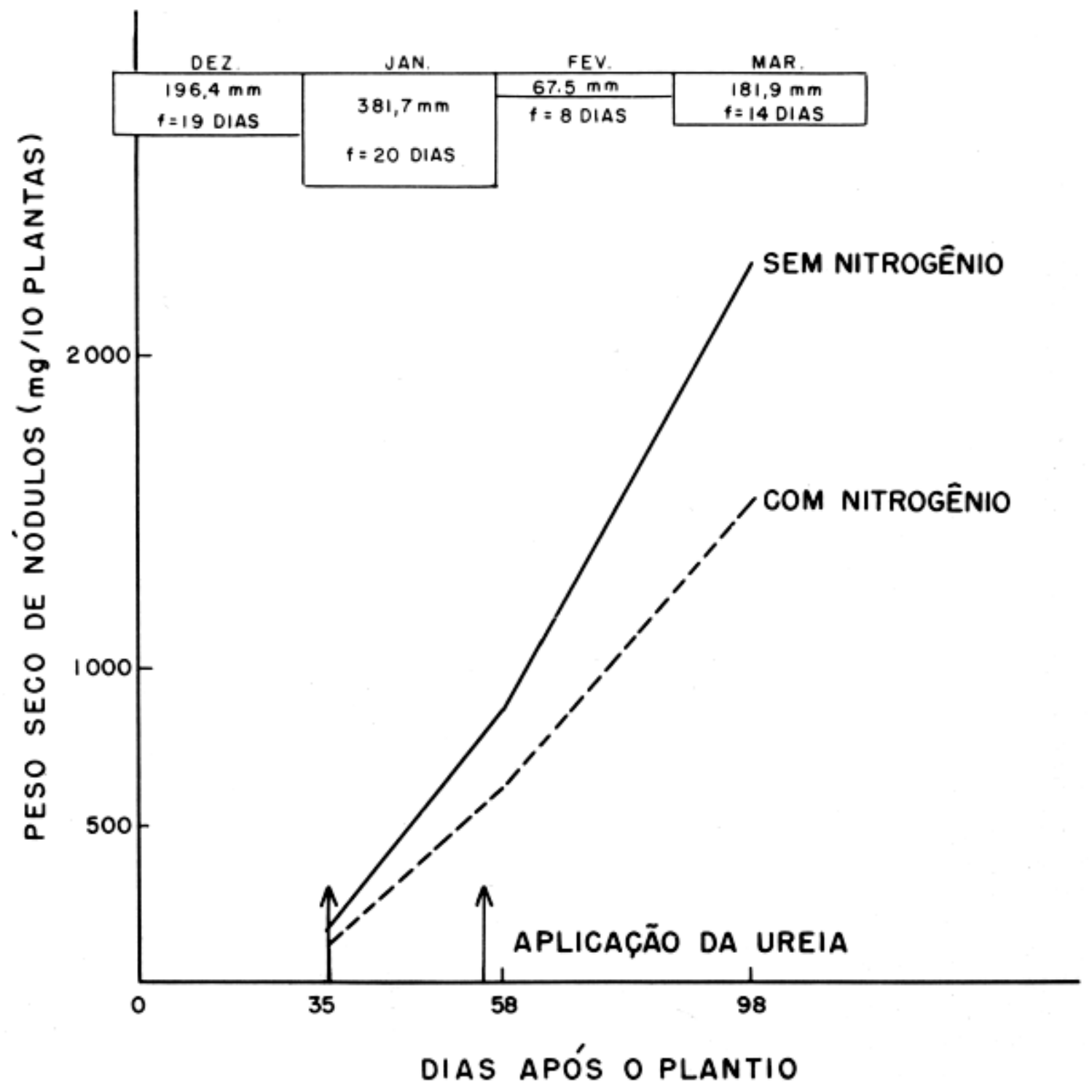

Figura 4. Influència do nitrogènio na nodulação da soja inoculada. Ano agrícola - 1973/1974. 
tenha ocorrido no presente ensaio, não houve consequências no seu desenvolvimento posterior da nodulação. A aplicação de alachlor em feijoeiro não prejudicou a nodulação dessa leguminosa, segundo Tovar e Mata (14), o que está de acordo com os resultados aqui obtidos com esse herbicida, para a soja.

$\mathrm{O}$ estudo da influência do nitrogênio na nodulação só pode ser efetuado a partir da segunda amostragem de nódulos, já que a aplicação da primeira

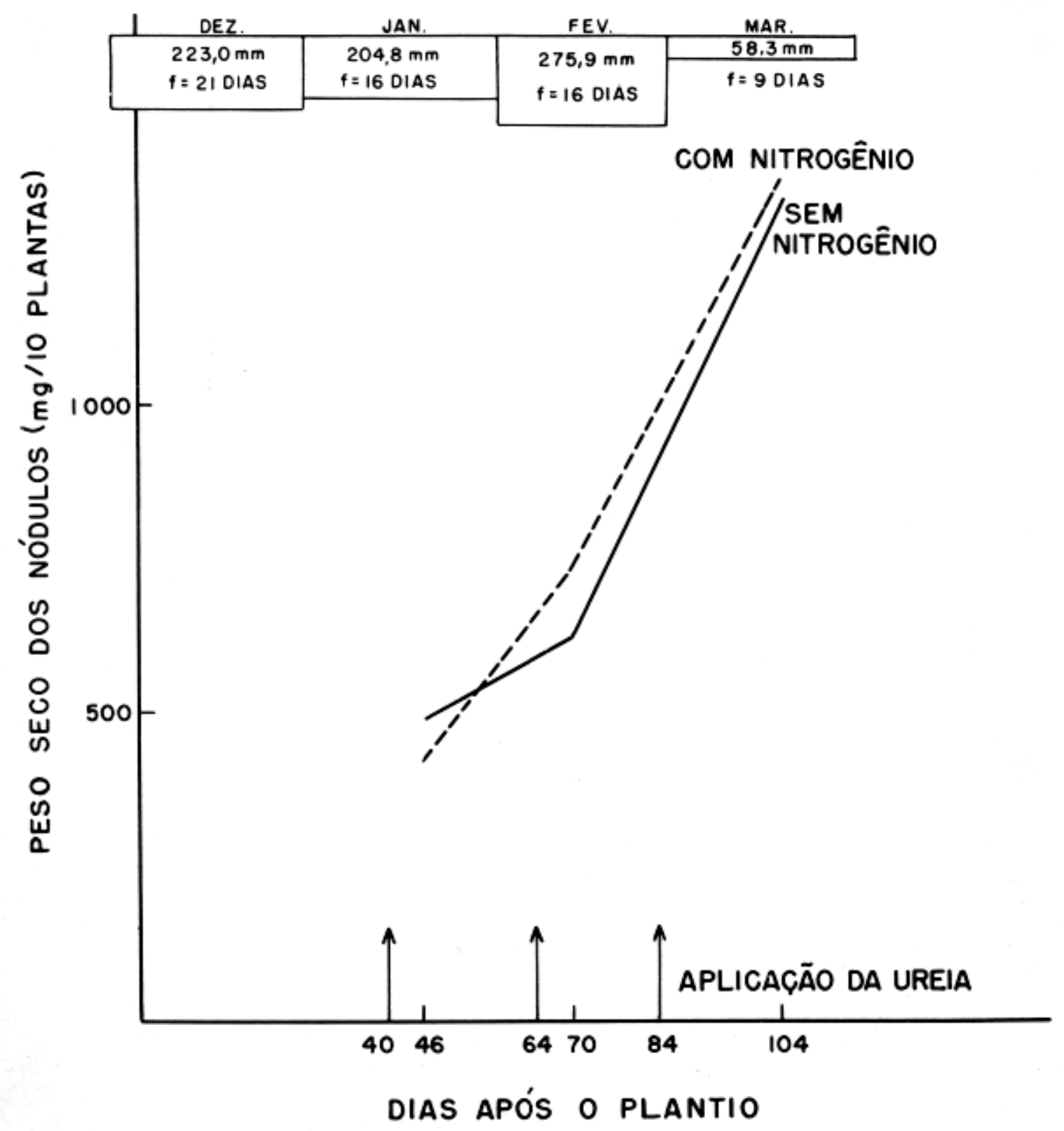

Figura 5. Influência do nitrogènio na nodulaçáo da soja inoculada. Ano agrícola - 1974/1975. 
parcela do adubo nitrogenado foi coincidente com a primeira amostragem de nódulos, no primeiro ano do ensaio, e aos seis dias antes da aplicação, no segundo ano. Os dados de número e peso de nódulos, observados nas três épocas de amostragens, para os tratamentos com e sem nitrogênio estão representados nas figuras 4 e 5 , para o primeiro e segundo ano, res pectivamente.

$\mathrm{Na}$ figura 4, observa-se que o nitrogênio da primeira aplicação (30 $\mathrm{kg} / \mathrm{ha}$ - 23 dias antes da amostragem de nódulos) fez diminuir, significativamente, o peso, mas não 0 número de nódulos observados os 58 dias. Na amostragem efetuada aos 98 dias $(30 \mathrm{~kg} \mathrm{~N} /$ ha aplicado 55 dias pósplantio) verificou-se que a aplicação do adubo nitrogenado diminuiu significativamente o peso e o número de nódulos. A diminuição da nodulação das leguminosas por efeito de nitrogênio combinado já é bem conhecida. Barni et al. (1) relatam redução significativa no peso de nódulos do cultivar Bragg em amostragens efetuadas na floração, com a aplicação de $64 \mathrm{~kg} \mathrm{~N} / \mathrm{ha}$, mas não com $32 \mathrm{~kg}$.

No segundo ano, a primeira amostragem de nódulos foi feita seis dias após a aplicação da primeira dose de nitrogênio. A análise estatística dos dados obtidos nos tratamentos inoculados revelou que não houve influên cia do nitrogênio, no número e peso de nódulos secos.

$\mathrm{Na}$ segunda amostragem de nódulos, aos 6 dias depois da segunda aplicação de nitrogênio, e na terceira amostragem, aos 20 dias após a última dose de nitrogênio, também não foram observadas diferenças significativas na nodulação. Entretanto, nos tratamentos sem inoculação (Figura 6), com nodulação espontânea, o peso seco dos nódulos foi significativamente maior nos tratamentos sem nitrogênio, nas três épocas amostradas.

B) Efeito dos tratamentos na produção de matéria seca das raízes e parte aérea:
Durante o primeiro ano do experimento, as médias gerais dos pesos de raizes secas por 10 plantas foram 3,23 g, $11,53 \mathrm{~g}$ e 23,80 g para amostragens efetuadas aos 35, 58 e 98 dias, respectivamente. Não foram constatados quaisquer efeitos significativos da inoculação, nitrogênio ou da aplicação dos herbicidas no peso das raízes, ou da parte aérea das plantas. Para a parte aérea, esses valores foram os seguintes: $16,60 \mathrm{~g}$, $69,69 \mathrm{~g}$ e $182,19 \mathrm{~g}$, respectivamente. No segundo ano, esses valores não foram determinados.

C) População de plantas e produção de sementes

Pela contagem das plantas por ocasião da colheita verificou-se que no primeiro ano os tratamentos de herbicida, inoculação ou adubação nitrogenada não influenciaram o "stand" final (quadro 1 e 2). A média de plantas estabelecidas neste ano foi de 18,5 por metro linear, para o experimento todo. No segundo ano, o "stand" final do experimento (quadro 1) foi mais baixo $(13,27$ plantas por metro) e o tratamento com inoculação das sementes apresentou um número significativamente menor de plantas (11,5 por metro) do que o tratamento sem inoculação $(15,1$ por metro). Não se pode adiantar uma explicação para essa observação, uma vez que, após a germinação, o "stand" foi padronizado para vinte plantas por metro, nos dois anos.

No quadro 1 estão as médias de produção por planta do $1 .^{\circ}$ e $2 .^{\circ}$ ano, dos tratamentos inoculados e não inoculados, em presença e ausência de nitrogênio, e no quadro 2 estão os dados de produções médias dos tratamentos com ou sem a aplicação dos herbicidas. No primeiro ano do experimento não foram observadas diferenças significativas na produção, quer por efeito da inoculação, do adubo nitrogenado, ou dos herbicidas aplicados. Como não houve nodulação nos tratamentos não inoculados (sem adição de nitrogênio), pode-se concluir que o suprimento de 


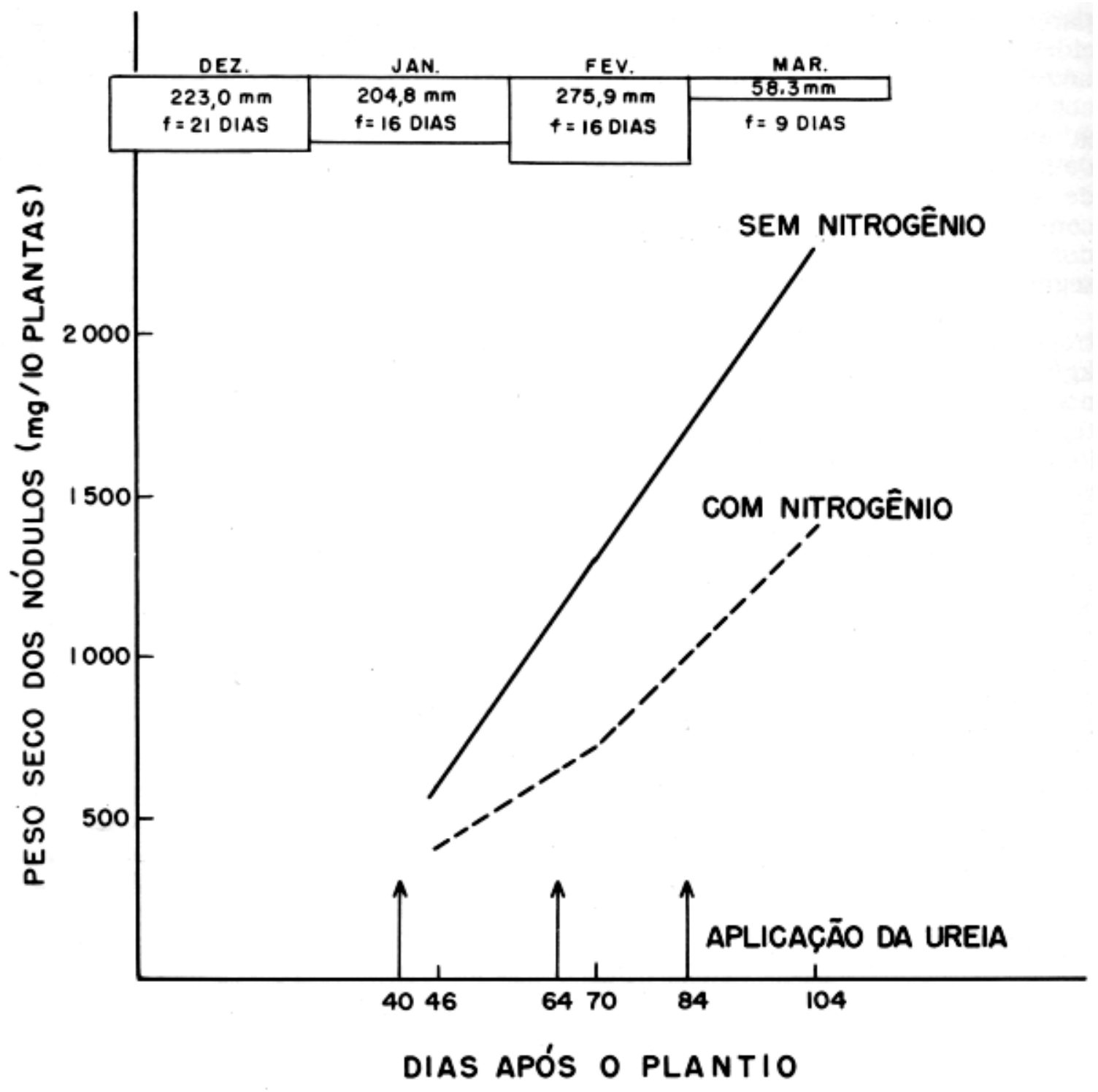

Figura 6. Influência do nitrogènio na nodulaçâo da soja não inoculada. Ano agrícola - 1974/1975.

nitrogênio do solo, neste primeiro ano foi satisfatório para a produção observada. A média geral de produção de sementes neste ano foi equivalente a $2.159 \mathrm{~kg} / \mathrm{ha}$.

No segundo ano, a média geral de produção de sementes foi bem menor que no ano anterior e equivalente a $1087 \mathrm{~kg} / \mathrm{ha}$. Convém ressaltar que, por causas não controladas, o "stand" final também foi menor neste ano, como já foi discutido.

Por outro lado; houve um efeito altamente significativo da inoculação, na ausência de nitrogênio, que foi equivalente a $82 \%$ de aumento na produção por planta. Na média dos tratamentos não inoculados ou inoculados, não houve aumento de produção devido ao adubo nitrogenado, indicando bom funcio- 
Quadro 1. Produção de sementes e número de plantas de soja com e sem inoculação ou adubação nitrogenada no experimento de herbicidas, em solo barrento, em Campinas (').

\begin{tabular}{|c|c|c|c|c|c|c|c|c|}
\hline \multirow{3}{*}{ TRATAMENTO } & \multicolumn{4}{|c|}{$1 .^{\circ}$ ANO } & \multicolumn{4}{|c|}{$2 .^{\circ}$ ANO } \\
\hline & \multicolumn{2}{|c|}{ SEM INOCULAÇÃO } & \multicolumn{2}{|c|}{ INOCULADO } & \multicolumn{2}{|c|}{ SEM INOCULAÇÃO } & \multicolumn{2}{|c|}{ INOCULADO } \\
\hline & n. ${ }^{\circ}$ & $\mathrm{g}$ & n. ${ }^{0}$ & $\mathrm{~g}$ & $\mathrm{n}^{\circ}$ & $\mathrm{g}$ & $\mathrm{n}^{\circ}$ & $\mathrm{g}$ \\
\hline Sem nitrogênio & 18,12 & 6,80 & 18,54 & 7,30 & 15,15 & 3,45 & 11,07 & 6,30 \\
\hline Com nitrogênio & 18,99 & 6,90 & 18,44 & 7,00 & 15,02 & 4,54 & 11,89 & 5,45 \\
\hline C.V. \% & \multicolumn{4}{|c|}{15} & \multicolumn{4}{|c|}{41} \\
\hline
\end{tabular}

(1) Pesos por planta e número por metro linear.

Média de 20 repetições ( 4 tratamentos com e sem herbicidas).

Quadro 2. Influência de herbicidas na produção de sementes e $n .^{\circ}$ de plantas de soja, em experimento conduzido em Campinas, em solo barrento, por dois anos consecutivos (1).

\begin{tabular}{|c|c|c|c|c|}
\hline \multirow[t]{2}{*}{ TRATAMENTO } & \multicolumn{2}{|c|}{$1 .^{\circ}$ ANO } & \multicolumn{2}{|c|}{$2 .^{\circ}$ ANO } \\
\hline & $\mathrm{n}^{0}$ & $\mathrm{~g}$ & $\mathrm{n}^{0}$ & g \\
\hline Sem herbicida & 18,93 & 6,5 & 12,99 & $3,18 \mathrm{~b}$ \\
\hline Com trifluralin & 18,72 & 7,0 & 13,12 & $6,23 a$ \\
\hline Com vernolate & 18,19 & 7,3 & 13,80 & $5,40 \mathrm{a}$ \\
\hline Com alachlor & 18,26 & 7,3 & 13,22 & $4,91 \mathrm{a}$ \\
\hline C.V. \% & \multicolumn{2}{|c|}{15} & \multicolumn{2}{|c|}{41} \\
\hline D.M.S. 5\% & \multicolumn{2}{|c|}{ ns } & \multicolumn{2}{|c|}{1,70} \\
\hline
\end{tabular}

(1) $\mathrm{N}^{\circ}$ de plantas por metro linear e pesos por planta.

Média de 20 repetições (4 tratamentos com e sem inoculação, com e sem nitrogênio).

namento da fixação simbiótica (quadro 1). É interessante ressaltar que os tratamentos não inoculados estavam também nodulados, o que sugere que essa nodulação não deve ter sido provocada pelas estirpes do inoculante, que tenham sobrevivido da inoculação do ano anterior. Um processo de adaptação de Rhizobium sp, do tipo "cow pea", em soja já foi sugerido (2). Neste experimento, não foi possivel comprovar essa hipótese, que, se ocorreu, não determinou sim- biose eficiente. Não foi também verificado efeito sinérgico do nitrogênio $(60 \mathrm{~kg}$ no primeiro ano e $90 \mathrm{~kg} / \mathrm{ha}$ no segundo ano) na produção de semente, como relatado por Ruschel e Ruschel (13) visto que as produções nos tratamentos inoculados, com e sem nitrogênio, foram estatisticamente semelhantes, tanto no primeiro como no segundo ano. Também foram semelhantes as produções dos tratamentos não inoculados na presença ou na ausência do nitrogênio. 
Neste segundo ano, as parcelas tratadas com herbicidas aumentaram significativamente a produção de sementes em relação às não tratadas; não é, entretanto, possivel atribuir esse efeito de aplicação dos herbicidas ao controle das plantas daninhas, já que os tratamentos sem aplicação dos herbicidas foram capinados.

\section{LITERA TURA CITADA}

1. Barni, N.A.; Kolling, J. \& Minor, H.C. Efeitos de niveis de nitrogênio sobre o rendimento de gráos, nodulaçáo e características agronómicas da soja (Glycine $\max$ L. Merr.). Agronomia Sulriograndense 11: 93-103, 1977.

2. Bonnier, C. \& Brakel, J. Lutte biologique contre la faim - legumineuses - rhizobium. Editions J. Duculot, S.A.Gembloux, 1969. 148pp.

3. Deuber, R. \& Forster, R. Influência do EPTC na nodulaçáo natural do feijáo (Phaseolus vulgaris L.). Planta Daninha 1(2): 38-43, 1978.

4. Kust, C.A. \& Struckmeyer, B.E. Effects of trifluralin on growth, nodulation and anatomy of soybeans. Weed Sei. 19: 147-152, 1971.

5. Lopes, E.S.; Deuber, R.; Forster, R.; Gargantini, H. \& Bulisani, E.A. Influência dos herbicidas EPTC e trifluralin e da inoculação das sementes com Rhizobium phaseoli na nodulação e produção do feijoeiro (Phaseolus vulgaris L.). Bragantia 30: 109-116, 1971.

6. Lopes, E.S.; Giardini, A.R.; Kilhl, R.A.S. \& Igue, T. Especificidade hospedeira e pré-seleçáo de estirpes de Rhizobium japonicum para as variedades santa rosa, viçoja e IAC-2, de soja (Glycine max L. Merril). Bragantia 35: 1-11, 1976.
7. Lopes, E.S.; Giardini, A.R.; Kiihl, R.A.S. \& Igue, T. Presença e eficiência de Rhizobium japonicum em solos cultivados ou não com soja, no Estado de São Paulo. Bragantia 35: 389-396, 1976.

8. Lorenzi, H.J. \& Araujo, S.C. Estudo da ação de alguns herbicidas na fixação simbiótica do nitrogênio em plantas de soja (Glycine $\max$ L. Merril). In: Reunion Latinoamericana sobre $R$ hizobium. $7 .^{\circ}$, Anais. Instituto Agrotécnico - Faculdade de Ciências Agrárias, Resistència, Argentina, pg.222-231, 1974.

9. Marriel, L.E. \& Cruz, J.C. Increased $\mathrm{N}_{2}$ fixation $\left(\mathrm{C}_{2} \mathrm{H}_{2}\right)$ in field grown maize by herbicide treatments. In: J. Dobbereiner; R.H. Burris e A. Hollaender, ed. Limitátions and potentials for biological nitrogen fixation in the tropics. Basic Life Seiences V. 10. Plenum Press, N. York. p.340, 1978.

0. Melfi, A.S.; Girardi, A.V. \& Moniz, A.C. Mineralogia dos solos da E.E. *Theodureto de Camargo* em Campinas. Bragantia 25: 930, 1966.

1. Parker, M.B. \& Dowler, C.C. Effects of nitrogen with trifluralin and vernolate on soy. beans. Weed Sci. 24: 131-133, 1976.

2. Phaik, Y.Y. \& Vincent, J.M. Influence of herbicides on the interaction between Rhizobia and host. In: Australian Legume Nodulation Conference, 5. C.S.I.R.O. Division of Tropical Agronomy. Queensland, Australia, Supplement to Rhizobium Newsletter 20: 87-90, 1975.

3. Ruschel, A.P. \& Ruschel, R. Sinergia da absorçáo de nitrogênio do solo e da fixaçāo simbiótica de nitrogènio atmosférico dirigido para o aumento do $\mathrm{N}$ total da soja (Glycine max). Pesq. Agrop. Bras. 10: 37-40, 1975.

4. Tovar, E.M. \& Mata, R.H. Efecto de mezcla de herbicidas en la nodulacion de ocho variedades de frijol (Phaseolus vulgaris L.) y algunas correlaciones entre los diferentes factores de crecimiento. El Agricultor Costarricence 5-6: 184-190, 1975. 